\section{ORIGINAL RESEARCH}

R.S. Pobiel

K.P. Schellhas

J.A. Eklund

M.J. Golden

B.A. Johnson

S. Chopra

P. Broadbent

M.E. Myers

K. Shrack

\title{
Selective Cervical Nerve Root Blockade: Prospective Study of Immediate and Longer Term Complications
}

\begin{abstract}
BACKGROUND AND PURPOSE: Selective cervical nerve root blockade (SCNRB) is a useful procedure for evaluating and treating patients with cervical radiculopathy. Reports of complications related to injections within the cervical nerve root foramen have raised serious doubts regarding the safety of this procedure. This study was performed to prospectively evaluate the safety of fluoroscopically guided outpatient diagnostic and therapeutic SCNRB.
\end{abstract}

\begin{abstract}
MATERIALS AND METHODS: Eight hundred two consecutive fluoroscopically guided diagnostic and/or therapeutic SCNRBs in 659 patients were performed during a 14-month period (November 2006 December 2007) at affiliated outpatient imaging centers. Each examination was performed by 1 of 8 experienced procedural radiologists by using an anterior oblique approach, with the needle position confirmed with radiographic contrast before injection of an admixture of local anesthetic and steroid. All patients were assessed immediately and at 30 minutes following the procedure. Additionally, 460 patients were called by telephone 30 days following the procedure. All complications were recorded.
\end{abstract}

RESULTS: Of the 802 attempted procedures, 799 were successfully completed. Three procedures were aborted due to anxiety, challenging body habitus, or persistent venous opacification observed during contrast injection and despite needle repositioning. There were no serious complications, such as stroke, spinal cord insult, permanent nerve root deficit, infection, or significant hematoma. There were 33 minor complications occurring within 30 minutes of the procedure; the most common was vasovagal symptoms. Three hundred forty-five patients were successfully contacted by telephone at 30 days postinjection, 9 of whom reported increased or new pain symptoms.

CONCLUSIONS: With our technique, fluoroscopically guided SCNRB is a safe outpatient procedure with a low immediate and delayed complication rate.

$\mathbf{T}$ ransforaminal selective cervical nerve root blockade (SC$\mathrm{NRB}$ ) is used as a diagnostic and/or therapeutic procedure in patients with cervical radiculopathy. A recent report retrospectively reviewed all SCNRBs performed at our institution during a 13-year period. ${ }^{1}$ In 4612 patients in whom particulate steroid solution was injected, there were 2 serious complications, both of which we believe to have been related to the steroid solution used, neither of which resulted in permanent neurologic or medical sequelae. Despite this excellent safety record, concerns about the safety of this procedure ${ }^{2}$ voiced in the literature and reports of serious complications prompted our group to evaluate SCNRB further in a prospective manner.

\section{Materials and Methods}

Data on all SCNRBs performed at our institution from November 2006 through December 2007 were collected with specific attention to complications or side effects of the procedure. Written consent to participate in research and informed consent for the procedure were obtained from all subjects. One patient declined to participate in the study and was excluded. Eight hundred two injections were attempted in 659 patients (age range, 25-89 years; median, 50 years; 414 men, 388 women) at 1 of 8 outpatient imaging centers in the Minneapo-

Received September 2, 2008; accepted after revision October 20.

From the Center for Diagnostic Imaging, St Louis Park, Minn.

Please address correspondence to Ronald S. Pobiel, MD, Center for Diagnostic Imaging, 5775 Wayzata Blvd, Ste 190, St Louis Park, MN 55416; e-mail: rpobiel@cdirad.com

Indicates open access to non-subscribers at www.ajnr.org

DOI 10.3174/ajnr.A1415
lis-St Paul metropolitan area (Center for Diagnostic Imaging, St Louis Park, Minn). Procedures were performed by 1 of 8 experienced procedural radiologists ( 7 formally trained neuroradiologists) engaged in the full-time practice of spine injection.

Procedures were performed to investigate the origins of suspected cervical radiculopathy, to treat radicular pain, or both. Patients were referred by physicians unaffiliated with our institution; specialties included orthopedic spine surgery, neurosurgery, neurology, physiatry, anesthesia-pain management, internal medicine, and family practice. One hundred forty-three patients had $>1$ procedure, 80 of whom had repeat or alternate levels injected on a separate day and 63 had an additional nerve blocked on the same day, typically with at least a 30-minute delay between injections.

The procedural technique has been recently described in detail. ${ }^{1}$ Briefly, all procedures were performed with high-resolution C-arm fluoroscopy, with patients in a supine position. Using intermittent fluoroscopy, a 25-gauge Quinke-type spinal needle (BD Medical Systems, Franklin Lakes, NJ) was advanced to the articular pillar, lateral to the posteroinferior aspect of the neural foramen. An anterolateral approach was performed to avoid the carotid and vertebral arteries. Following contact of the pillar or provocation of radicular pain, iohexol $240 \mathrm{mg} / \mathrm{mL}$ (Omnipaque; GE Healthcare, Princeton, NJ) was injected to confirm the needle position. In 2 patients, MR imaging gadolinium contrast agent (gadodiamide, Omniscan; GE Healthcare; or gadopentetate dimeglumine, Magnevist; Bayer HealthCare, Wayne, NJ) was used secondary to a history of severe iodinated contrast allergy. Typically, 1-2 mL of contrast was used, and the needle was carefully adjusted until an adequate neurogram was obtained (Fig 1). Following filming to document needle position, $1.0-1.6 \mathrm{~mL}$ total of a 1:2 or 1:3 admixture of steroid-lidocaine $2 \%$ was instilled. The 




Fig 1. Selective right C4 nerve root blockade. Anteroposterior projection showing an optimum neurogram.

steroid used was betamethasone sodium phosphate/acetate suspension (Celestone Soluspan; Schering, Kenilworth, NJ), methylprednisolone acetate $40 \mathrm{mg} / \mathrm{mL}$ (Depo-Medrol; Pfizer Pharmacia and Upjohn Co, New York, NY), or dexamethasone sodium phosphate 10 $\mathrm{mg} / \mathrm{mL}$ (Decadron; Abraxis, Schaumberg, Ill). Approximately 6 months into the study, we discontinued using particulate steroids in favor of nonparticulate dexamethasone sodium phosphate. Procedure fluoroscopy time was recorded.

Thirteen procedures were performed with conscious sedation. In these cases, the procedure was performed in a Medicare-certified ambulatory surgery center under close physiologic monitoring. The patients were typically given 1 - to 2 -mg midazolam and 25- to $100-\mu \mathrm{g}$ fentanyl citrate (Hospira, Lake Forest, Ill) and monitored at least 1 hour following administration of medication.

Patients were assessed for immediate procedural complications. All unexpected symptoms or signs were evaluated and documented by the procedural physician. Patients were placed in a waiting area for observation and reassessed in 30 minutes by the procedural physician (in most cases) or by an experienced procedural nurse or technologist. Assessment included the general well being of the patient, gait, papillary or vision changes, dizziness, facial and extremity muscle weakness, and new sensory symptoms or deficits. Patients were asked to rate their response to the injection on either a $0-10$ visual analog scale (VAS) or as a percentage of pain relief. Any significant complication was duly recorded, and the patient was retained for additional observation and was discharged by the procedural physician. Patients were instructed to call our 24-hour clinic line during the ensuing week for any questions or concerns related to the procedure and for any swelling, local redness, increased pain, fever, or new extremity weakness. Telephone follow-up at 30 days postprocedure was attempted by an experienced procedural nurse in 460 cases. Successful contact was made in 345 cases. All problems or comments related to the procedure were recorded. Patients were asked about new neurologic symptoms and improvement or worsening of previous symptoms.

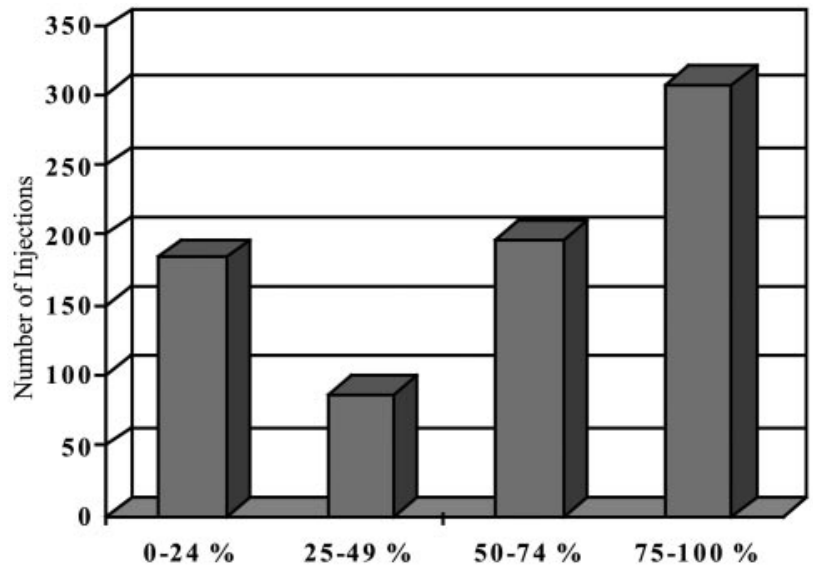

Percent Pain Relief 30 Minutes Post Injection

Fig 2. Graph shows 30-minute pain response by quartile.

\section{Minor complications or events}

\begin{tabular}{lc}
\hline Event & No. \\
Patients
\end{tabular}

\section{Results}

Seven hundred ninety-nine injections were technically successful. There were 50 injections at the $\mathrm{C} 4$ nerve root, 112 at C5, 300 at C6, 305 at C7, and 32 at C8. The average fluoroscopy time was 32.2 seconds (range, 5-214 seconds). There were 3 aborted procedures. One patient could not hold still due to anxiety during attempted C7 nerve root blockade, and this study was electively aborted without complication. This patient was subsequently successfully injected under conscious sedation. Another patient (C8 nerve root) was technically challenging because of the body habitus (short neck and kyphosis) and his inability to refrain from swallowing during needle placement. After discussion with the patient and referring physician, he underwent a successful therapeutic interlaminar cervical epidural steroid injection as an alternative. A third patient had an unsuccessful C5 SCNRB due to persistent venous opacification at the $\mathrm{C} 4-5$ foramen, despite multiple needle repositioning maneuvers. This patient had had prior C5-6 and C6-7 anterior diskectomy and interbody fusion.

Thirty-minute initial pain response to anesthetic is summarized by quartile in Fig 2 . There were no major or permanent complications. There were $40(5.0 \%)$ events reflecting minor complications, potential complications, or side effects (Table).

Thirty-three of these events occurred within 30 minutes of the procedure, and 7 were discovered at the 30-day follow-up. Early events included 19 vasovagal-like episodes, typically experienced as nausea, diaphoresis, and/or lightheadedness during or immediately after the procedure. All of these events 
responded to conservative measures such as Trendelenburg positioning, cool compresses, or oral liquids. There were 6 patients with transient sympathetic blockade, 4 following C7 and 2 following C6 SCNRB. Four of these patients had transient unilateral ptosis and miosis following C7 blockade, which gradually resolved without sequelae. One of these patients also had transient conjunctiva redness. Two patients had sympathetic blockade following C6 blockade. One had mild miosis, ptosis, and subjective blurred vision, which resolved. This patient had a history of ipsilateral carotid endarterectomy.

A second patient had sudden blurred vision and transient conjuctiva redness following left C6 blockade. The diagnosis of anesthetic-related sympathetic blockade was confirmed by an ophthalmologist the following day and symptoms resolved completely. Three patients had an initial increase in their typical pain pattern. One patient ultimately had good neck pain relief at 30-day follow-up. Another had a 30-minute 95\% relief response but increased pain after leaving the clinic. The third patient reported no relief initially or at 30 days. There was 1 patient with persistent nausea, which was completely resolved by the following morning. One patient had a clinically suspected local hematoma, which resolved without sequelae. There was 1 minor suspected contrast allergy (eyelid swelling and erythema). There was 1 case of new contralateral paresthesias following a C5 block. This symptom commenced approximately 3 weeks following the nerve block and was not thought to be related to the procedure. One patient reported a sensation of incomplete right lung expansion 10 minutes after an uneventful C5 nerve root block. Vital signs, pulse oximetry, and physical examination findings were normal, and the sensation resolved shortly thereafter.

Of the 345 patients contacted at the 30-day telephone follow-up, 30 had interval (previously planned) surgery. Of the remaining 315 patients, 7 reported increased pain in a familiar distribution. Of this 7,2 had complete resolution at 60-day telephone contact, 3 had subsequent surgery, and 2 were lost to follow-up. There were 2 patients with new pain symptoms that could not be attributed to the injection. One hundred sixty patients reported no change in baseline pain at 30 days. One hundred forty-eight patients (47\% of nonsurgical) reported pain improvement. For the improved group, the average VAS was 5.4 before injection and 2.2 (by telephone) at 30 days postinjection for an average VAS reduction of $59 \%$. The improved group reported an average 30-minute postinjection VAS reduction of 3.01 compared with a reduction of 2.98 in the 167 patients with no change or worsened symptoms at 30 days.

\section{Discussion}

Cervical radicular pain affects approximately 0.8 in 1000 persons per year, with the rate as high as 2 in 1000 per year in middle-aged groups. ${ }^{3}$ The typical etiology is cervical degenerative disease or spondylosis resulting in foraminal stenosis, with acute soft-disk herniation less common. Trauma or physical exertion is an antecedent event in a minority of patients$15 \%$ in 1 large population-based study. ${ }^{3}$ Although many patients improve with conservative therapy, a significant number of patients require surgery or develop chronic and sometimes debilitating symptoms. In these patients, cortico- steroid injection therapy may be used to deliver anti-inflammatory medication adjacent to or near the suspected site of pain generation. In addition to anti-inflammatory effects, corticosteroids may also decrease pain by stabilizing neural membranes and by a direct anesthetic effect on small unmyelinated nociceptive $\mathrm{C}$ fibers. ${ }^{4}$ Additionally, lidocaine has anti-inflammatory effects and may improve blood flow and reduce neural dysfunction in injured nerve roots. ${ }^{4}$

Medication may be delivered nonspecifically via an interlaminar or translaminar epidural steroid injection or may be deposited directly along the nerve root, as a selective nerve root block or transforaminal epidural injection. The addition of local anesthetic with limited injectant volume allows the selective nerve block to function as a diagnostic test, which is particularly useful in cases of multilevel spondylosis. The clinical usefulness and efficacy of the procedure has been supported by a number of prospective observational studies. ${ }^{4-9}$ In addition, studies have supported the relevance of selective nerve root blockade to clinical and MR imaging findings. ${ }^{10,11}$

Various techniques have been described for the transforaminal or selective cervical nerve block, but there is agreement that image guidance is imperative for both accuracy and safety. ${ }^{1,12-17}$ Imaging techniques include CT, ${ }^{14,15,18}$ CT fluoroscopy, ${ }^{19,20}$ and fluoroscopy. ${ }^{1,16}$ The advantage of CT guidance is the ability to visualize directly relevant soft-tissue structures, such as the carotid and vertebral arteries and to plan the needle trajectory accordingly. The advantage of fluoroscopy is the ability to see contrast dispersion in real-time with a broad FOV. We strongly believe that real-time visualization of the contrast injection is critical; therefore, we exclusively use fluoroscopy for all nerve blocks and transforaminal epidural injections. We also prefer and exclusively use the anterolateral over the true lateral needle approach, by using the articular pillar as a backstop for depth control and for avoiding the vertebral artery. Our desired needle position is just lateral to the posterior aspect of the neural foramen (extraforaminal approach). We typically avoid transforaminal dispersal of contrast with SCNRB and do not routinely perform transforaminal cervical epidural steroid injections. The posterior aspect of the lateral foramen is considered safest with regard to potential radicular artery branches; however, anatomic studies have concluded that radicular feeding arteries may be found at any aspect of the cervical foramen, ${ }^{21,22}$ supporting the need for real-time observation of contrast injection.

When performed with rigorous technique and by experienced operators, SCNRB is a safe technique, with a low rate of serious complications. ${ }^{1,17,23,24}$ Although uncommon, complications of SCNRB or transforaminal cervical epidural injections can be devastating. ${ }^{25-35}$ Reported major complications include spinal cord and/or brain infarction and death. Direct vascular penetration with intra-arterial injection of anesthetic and particulate steroid, vascular injury with dissection and thrombosis, or direct nerve root or spinal cord injury from the needle or injectant are postulated mechanisms of injury. Recent experimental work supports the role of particulate steroid injection in some cases of cord or brain infarction.

Okubadejo et $\mathrm{al}^{36}$ injected the vertebral artery of 11 pigs with different steroid preparations and examined the brains and cervical spinal cords by MR imaging and histology. Four pigs received particulate steroid (methylprednisolone, $40 \mathrm{mg}$ / 
$\mathrm{mL})$. All of these pigs failed to regain consciousness, and all demonstrated upper cervical cord and brain stem edema on MR imaging 4 hours postinjection. All 4 pigs demonstrated histologic findings of early neuronal necrosis. Seven pigs received water soluble steroid ( 3 prednisolone and 4 dexamethasone). All of these pigs fully regained consciousness without evidence of neurologic injury, had normal MR imaging the day following injection, and had normal histopathologic findings. In response to these data and despite our experience with $>5000$ SCNRBs by using particulate steroid, we have switched to a nonparticulate steroid (dexamethasone). Some authors advocate injecting anesthetic first, followed by steroid after a short delay to assess neurologic deficit. ${ }^{29,30,37,38}$ Our data with SCNRB and $>15,000$ transforaminal lumbar nerve injections support the safety of admixing local anesthetic and particulate steroid. This technique decreases the total procedure time, reducing the risk of inadvertent needle movement. Digital subtraction fluoroscopy $y^{25}$ and the use of microbore tubing to reduce needle manipulation ${ }^{29}$ have been advocated by some, though we do not routinely use either technique

Minor complications or side effects occurred in 5\% of injections in this series. None resulted in permanent sequelae, though 2 patients were lost to follow-up. There was no relationship of minor complications to the type of steroid used. Most minor complications were self-limited and not unique to SCNRB. For example, 19 patients had mild vasovagal-like symptoms, which responded promptly to conservative measures. This is consistent with the rate of such reactions in other spinal injections we perform and does not indicate an increased risk of the SCNRB procedure. Transient sympathetic blockade in 6 patients was due to the spread of anesthetic to nearby sympathetic branches or plexuses and may be rightly considered a side effect, rather than a complication of the procedure. The symptoms are similar to those seen with stellate ganglion blockade. The symptoms, transient clinical course, and lack of neck tenderness ruled out carotid dissection. Several patients noted increased or new pain symptoms following the procedure. This raised the possibility of nerve root trauma or radiculitis related to contrast or medication; however, the natural history of the disease may also be implicated. In a study of 89 cervical and 217 lumbar nerve root injections in 151 patients, Huston et $\mathrm{al}^{24}$ reported that $37 \%$ of patients had increased spine or radicular pain at 1 week postinjection. However, a control group with a similar diagnosis but who had not yet received injection therapy reported a statistically similar $34 \%$ rate of increased pain at 1 week.

Data are presented on both the initial 30-minute pain response in all study patients and the 30-day pain level in 315 of the 345 patients who were contacted by telephone and who did not have interval surgery. Although this study was not designed to evaluate the clinical outcome or efficacy of SCNRB, interesting points may be made. Most referrals to our practice for SCNRB are for diagnostic evaluation of chronic neck and radicular symptoms. Figure 2 demonstrates that most injections $(62 \%)$ produced strongly diagnostic responses, either in the $0 \%-24 \%$ or $75 \%-100 \%$ pain relief quartiles. Although initial pain response must be corroborated clinically and with imaging, this response suggests that the procedure may have diagnostic value in most patients. One factor that may decrease response reliability is the type of radicular symptom:
Extremity pain symptoms are more reliably evaluated with local anesthetic than numbness or tingling. At 30 days, $47 \%$ of patients reported pain improvement, with a mean VAS reduction of 59\%. Although uncontrolled, this finding may support the routine use of corticosteroids for SCNRB. Finally, there was no significant difference in mean 30-minute VAS reduction between patients who did and those who did not report pain improvement at 30 days. One could not predict at 30 minutes which patients would achieve some degree of pain relief at 30 days.

Our study has several limitations. There was no control group to evaluate the significance of the minor complications adequately, but the low rate and clinical insignificance of these complications probably does not warrant inclusion of a control group. All patients were not contacted by our nurse at 30 days. Twenty-five percent of attempted calls were unsuccessful, and there was not enough staff to attempt to call all patients at 30 days. It is likely that additional minor complications or events would have been detected had all patients been contacted at 30 days. We do not believe that this is a significant limitation, however, because serious complications from SCNRB typically occur immediately or shortly after the procedure and all patients were thoroughly evaluated during this timeframe. The data may be biased by lack of an independent physician, such as a neurologist, evaluating the patients following injection. In addition, the patients did not receive a formal neurologic examination following the procedure. Although large, the number of procedures included in this study may be too small to pick up an uncommon major complication such as spinal cord or brain infarction. In our recent retrospective study of 4612 patients undergoing the same technique with particulate steroid, there were no permanent serious neurologic events. ${ }^{1}$ Since termination of the study, we have performed over 600 additional procedures with no major immediate complication.

\section{Conclusions}

When performed by experienced proceduralists using fluoroscopic guidance and meticulous technique, our described SCNRB is a safe procedure with an extremely low incidence of serious and minor complications.

\section{References}

1. Schellhas KP, Pollei SR, Johnson BA, et al. Selective cervical nerve root blockade: experience with a safe and reliable technique using an anterolateral approach for needle placement. AJNR Am J Neuroradiol 2007;28:1909-14

2. Santiago-Palma J, Vallejo R, Kornick C, et al. Are cervical nerve root blocks "safe and effective"? AJNR Am J Neuroradiol 2005;26:2434-35

3. Radhakrishnan K, Litchy WJ, O'Fallon WM, et al. Epidemiology of cervical radiculopathy: a population-based study from Rochester, Minnesota, 1976 through 1990. Brain 1994;117(part 2):325-35

4. Slipman CW, Lipetz JS, Jackson HB, et al. Therapeutic selective nerve roo block in the nonsurgical treatment of atraumatic cervical spondylotic radicular pain: a retrospective analysis with independent clinical review. Arch Phys Med Rehabil 2000;81:741-46

5. Cyteval C, Thomas E, Decoux E, et al. Cervical radiculopathy: open study on percutaneous periradicular foraminal steroid infiltration performed under CT control in 30 patients. AJNR Am J Neuroradiol 2004;25:441-45

6. Vallée JN, Feydy A, Carlier RY, et al. Chronic cervical radiculopathy: lateralapproach periradicular corticosteroid injection. Radiology 2001;218:886-92

7. Kolstad F, Leivseth G, Nygaard OP. Transforaminal steroid injections in the treatment of cervical radiculopathy: a prospective outcome study. Acta Neurochir (Wien) 2005;147:1065-70. Epub 2005 Jun 9

8. Razzaq AA, O'Brien D, Mathew B, et al. Efficacy and durability of fluoroscopically guided cervical nerve root block. Br J Neurosurg 2007;21:365-69 
9. Bush K, Hillier S. Outcome of cervical radiculopathy treated with periradicular/epidural corticosteroid injections: a prospective study with independent clinical review. Eur Spine J 1996;5:319-25

10. Anderberg L, Annertz M, Brandt L, et al. Selective diagnostic cervical nerve root block: correlation with clinical symptoms and MRI-pathology. Acta Neurochir (Wien) 2004;146:559-65. Epub 2004 Apr 26

11. Sasso RC, Macadaeg K, Nordmann D, et al. Selective nerve root injections can predict surgical outcome for lumbar and cervical radiculopathy: comparison to magnetic resonance imaging. J Spinal Disord Tech 2005;18:471-78

12. Wagner AL, Murtagh FR. Selective nerve root blocks. Tech Vasc Interv Radiol 2002;5:194-200

13. Rathmell JP, Aprill C, Bogduk N. Cervical transforaminal injection of steroids. Anesthesiology 2004;100:1595-600

14. Zennaro $\mathrm{H}$, Dousset $\mathrm{V}$, Viaud $\mathrm{B}$, et al. Periganglionic foraminal steroid injections performed under CT control. AJNR Am J Neuroradiol 1998;19:349-52

15. Wagner AL. CT fluoroscopic-guided cervical nerve root blocks. AJNR Am J Neuroradiol 2005;26:43-44

16. Gilula LA, Ma D. A cervical nerve block approach to improve safety. AJR Am J Roentgenol 2007;189:563-65

17. Ma DJ, Gilula LA, Riew KD. Complications of fluoroscopically guided extraforaminal cervical nerve blocks: an analysis of 1036 injections. J Bone Joint Surg Am 2005;87:1025-30

18. Quinn SF, Murtagh FR, Chatfield R, et al. CT-guided nerve root block and ablation. AJR Am J Roentgenol 1988;151:1213-16

19. Kim H, Lee SH, Kim MH. Multislice CT fluoroscopy-assisted cervical transforaminal injection of steroids: technical note. J Spinal Disord Tech 2007;20:456-61

20. Bartynski WS, Whitt DS, Sheetz MA, et al. Lower cervical nerve root block using CT fluoroscopy in patients with large body habitus: another benefit of the swimmer's position. AJNR Am J Neuroradiol 2007;28:706-08

21. Huntoon MA. Anatomy of the cervical intervertebral foramina: vulnerable arteries and ischemic neurologic injuries after transforaminal epidural injections. Pain 2005;117:104-11

22. Hoeft MA, Rathmell JP, Monsey RD, et al. Cervical transforaminal injection and the radicular artery: variation in anatomical location within the cervical intervertebral foramina. Reg Anesth Pain Med 2006;31:270-74

23. Derby R, Lee SH, Kim BJ, et al. Complications following cervical epidural steroid injections by expert interventionalists in 2003. Pain Physician 2004;7:445-49

24. Huston CW, Slipman CW, Garvin C. Complications and side effects of cervical and lumbosacral selective nerve root injections. Arch Phys Med Rehabil 2005;86:277-83

25. Beckman WA, Mendez RJ, Paine GF, et al. Cerebellar herniation after cervical transforaminal epidural injection. Reg Anesth Pain Med 2006;31:282-85

26. Brouwers PJ, Kottink EJ, Simon MA, et al. A cervical anterior spinal artery syndrome after diagnostic blockade of the right C6-nerve root. Pain 2001;91:397-99

27. McMillan MR, Crumpton C. Cortical blindness and neurologic injury complicating cervical transforaminal injection for cervical radiculopathy. Anesthesiology 2003;99:509-11

28. Rozin L, Rozin R, Koehler SA, et al. Death during transforaminal epidural steroid nerve root block (C7) due to perforation of the left vertebral artery. Am J Forensic Med Pathol 2003;24:351-55

29. Tiso RL. Adverse central nervous system sequelae after selective trasforaminal block. Spine J 2006;6:219

30. Scanlon GC, Moeller-Bertram T, Romanowsky SM, et al. Cervical transforaminal epidural steroid injections: more dangerous than we think? Spine 2007;32:1249-56

31. Wallace MA, Fukui MB, Williams RL, et al. Complications of cervical selective nerve root blocks performed with fluoroscopic guidance. AJR Am J Roentgenol 2007; 188:1218-21

32. Ludwig MA, Burns SP. Spinal cord infarction following cervical transforaminal epidural injection: a case report. Spine 2005;30:E266-68

33. Windsor RE, Storm S, Sugar R, et al. Cervical transforaminal injection: review of the literature, complications, and a suggested technique. Pain Physician 2003;6:457-65

34. Muro K, O'Shaughnessy B, Ganju A. Infarction of the cervical spinal cord following multilevel transforaminal epidural steroid injection: case report and review of the literature. J Spinal Cord Med 2007;30:385-88

35. Rosenkranz M, Grzyska U, Niesen W, et al. Anterior spinal artery syndrome following periradicular cervical nerve root therapy. J Neurol 2004;251:229-31

36. Okubadejo G, Talcott M, Schmidt R, et al. Perils of intravascular methylprednisolone injection into the vertebral artery: an animal study. J Bone Joint Surg Am 2008;90:1932-38

37. Karasek M, Bogduk N. Temporary neurologic deficit after cervical transforaminal injection of local anesthetic. Pain Med 2004;5:202-05

38. Baker R, Dreyfuss P, Mercer S, et al. Cervical transforaminal injection of corticosteroids into a radicular artery: a possible mechanism for spinal cord injury. Pain 2003;103:211-15 